\title{
Effects of Beta-Lactam Antibiotics and N-Methyltetrazolethiol on the Alcohol-Metabolizing System in Rats
}

\author{
Takashi MATSUBARA, Shigemi OTSUBO, Ayako OGAWA, \\ Keiko KAWAMOTO, Joji OKAMOTO, Koichi SUGENO, \\ Yoshihiro TOCHINO, Tadashi YOSHIDA and Eizo HIRAI
}

Shionogi Research Laboratories. Shionogi \& Co. Ltd.,

Fukushima-ku, Osaka 553, Japán

Accepted July 27. 1987

\begin{abstract}
The disulfiram-like effect of various beta-lactam antibiotics containing $\mathrm{N}$-methyltetrazolethiol (NMTT) on the alcohol-metabolizing system was studied using rats. Their administration caused decreased activities in low $\mathrm{K}_{\mathrm{m}}$ aldehyde dehydrogenase (ALDH) and acetaldehyde oxidation in the liver, with marked depression from several hours to 2 days after the treatment. Blood acetaldehyde level increased markedly when ethanol was administered 18-24 hr after pretreatment with antibiotics. A similar time course change in the effect was obtained when disulfiram was administered. The following results obtained in the present study indicate that the disulfiram-like effect associated with these antibiotics was not mediated by the whole molecular structures of these drugs: Firstly, the antibiotics were eliminated rapidly from the plasma and liver, and the disulfiramlike effect was followed by a disappearance of the drugs. Secondly, the concentration of antibiotics required to inhibit mitochondrial low $\mathrm{K}_{m}$ ALDH activity in vitro was very high compared with their liver concentration. Thirdly, rapid onset of disulfiram-like effects occurred after administration of NMTT itself, and a pronounced elevation of blood acetaldehyde level was observed when ethanol was administered $3-5 \mathrm{hr}$ after the NMTT injection. Fourthly, almost the same amounts of NMTT were released in the body after the intravenous administration of various NMTT-containing antibiotics, as judged by the urinary excretion. These results suggest that the disulfiram-like effect of beta-lactam antibiotics is mediated by NMTT released from them.
\end{abstract}

Several drugs have been reported to induce disulfiram-like effects in patients. who took an alcohol-containing drink while on these drugs (1). The disulfiram-like reaction or the disulfiram-alcohol interaction typically consists of marked flushing, headache, nausea and vomiting, and begins in the disulfiram-pretreated individual within a few minutes after ethanol intake (1). Disulfiram-like reactions have also been found in subjects receiving some beta-lactam antibiotics (2-6). Buening et al. (3, 4) and Yanagihara et al. $(7,8)$ have reported that some beta-lactam antibiotics, having the $\mathrm{N}$ methyltetrazolylthiomethyl group at the 3-position of the cephalosporin nucleus, increased the blood acetaldehyde concentration during ethanol metabolism in rats. Subsequent studies demonstrated that the administration of these antibiotics caused a decrease of liver mitochondrial low $\mathrm{K}_{\mathrm{m}}$ aldehyde dehydrogenase (ALDH) activity in rats $(9,10)$. Thus, these antibiotics are supposed to depress the low $K_{m}$ ALDH activity in liver mitochondria followed by an increase in the blood acetaldehyde level during ethanol metabolism (10).

Although the $\mathrm{N}$-methyltetrazolethiol (1methyl-1H-tetrazole-5-thiol. NMTT) is a common substituent at the $3^{\prime}$-position of the cephalosporin nucleus in the beta-lactam antibiotics producing the disulfiram-like ef- 
fects, it is hard to conclude which molecular structure causes the disulfiram-like reaction: i.e., whether the whole molecular structure of the antibiotic or the NMTT liberated from it is involved remains obscure. Thus, we examined the effect of beta-lactam antibiotics and NMTT itself on the acetaldehydemetabolizing system in rats, associated with the pharmacokinetic profile of the antibiotics. We found evidence that NMTT liberated from the antibiotics affects the acetaldehydemetabolizing system (IOW $K_{\mathrm{ra}} A L D H$ ) and increases the blood acetaldehyde concentration during the ethanol metabolism.

\section{Materials and Methods}

Animals: All experiments were performed using Slc Wistar strain male rats ( 9 weeks old). The animals were kept in an air conditioned room $\left(25 \pm 1^{\circ} \mathrm{C}\right.$. $50-60 \%$ humidity) lighted $12 \mathrm{hr}$ a day (8:00-20:00) and maintained on commercial rat chow (CA-1. Clea Japan Inc., Tokyo) and water ad libitum. All animals were allowed at least 7 days to become acclimatized to the housing conditions prior to use in the experiments.

Agents used: Beta-lactam antibiotics used for the experiments were as follows: cefamandole (CMD) and latamoxef or moxalactam (LMOX) were obtained from Shionogi \& Co. (Osaka), cefoperazone (CPZ) from Toyama Chemical Co. (Tokyo). cefmetazole (CMZ) from Sankyo Co. (Tokyo), cefotiam (CTM) and cefmenoxime (CMX) from Takeda Chemical Industries (Osaka), cefotetan (CTT) from Yamanouchi Pharmaceutical Co. (Tokyo) and cefazolin (CEZ) from Fujisawa Pharmaceutical Co. (Osaka). Unless otherwise specified, each antibiotic was dissolved in distilled water and administered subcutaneously at 1.000 $\mathrm{mg} / \mathrm{kg}$ body weight. Disulfiram was suspended in 5\% ( $w / v)$ arabic gum and administered orally at a dose of $1,000 \mathrm{mg} / \mathrm{kg}$. The sodium salt of NMTT was dissolved in distilled water and the resulting solution was administered subcutaneously at a dose of 300 or $500 \mathrm{mg} \mathrm{NMTT} / \mathrm{kg}$ (450 or $750 \mathrm{mg}$ NMTT-Na/kg).

Preparation of enzyme samples: The animals were killed by decapitation and their livers were removed quickly. Subcellular fractions of liver cells were prepared as described previously (10). The protein concentration of subcellular fractions was determined by the method of Lowry et al. (11) using bovine serum albumin as a standard.

Determination of enzyme activities: Aldehyde dehydrogenase (ALDH) activity was determined spectrophotometrically according to the method of Hasumura et al. (12) with slight modifications as described previously (10). After the initiation of the reaction by adding substrate, acetaldehyde, the formation of NADH from NAD was monitored at $340 \mathrm{~nm}$, and the ALDH activity was calculated from the initial velocity of the NADH formation. The acetaldehyde oxidation activity was determined by measuring the decreased amounts of acetaldehyde gas chromatographically (10), and alcohol dehydrogenase was detected as described previously (13). When disulfiram and its related compound were added to the in vitro reaction mixture, they were dissolved in $50 \%(\mathrm{v} / \mathrm{v})$ acetone, and an aliquot $10.05-$ $0.1 \mathrm{ml}$ ) of the resulting solution was added to the mixture $(3.0 \mathrm{ml}$ in total). Beta-lactam antibiotics were dissolved in distilled water and added as above.

Determination of ethanol and acetaldehyde concentrations in blood: Rats, fasted overnight. were given orally a $20 \%(\mathrm{~W} / \mathrm{v})$ solution of ethanol in water at a volume of 1.0 $\mathrm{ml} / 100 \mathrm{~g}$ body weight (2.000 $\mathrm{mg}$ ethanol/ $\mathrm{kg}$ ). Blood samples from decapitated rats were collected in heparin-containing tubes $1 \mathrm{hr}$ after the ethanol treatment. Heparinized blood $(4 \mathrm{ml})$ was mixed with $4 \mathrm{ml}$ of icecold $\mathrm{H}_{2} \mathrm{O}$ followed by $4 \mathrm{ml}$ of ice-cold $1.2 \mathrm{~N}$ perchloric acid. After centrifugation at about $2.000 \times \mathrm{g}$ for $15 \mathrm{~min}$, deproteinized supernatant $(1.5 \mathrm{ml})$ was transferred to a $15-\mathrm{ml}$ glass vial fitted with an airtight rubber septum and further capped with an aluminum seal. Ethanol and acetaldehyde concentrations in the vials were determined by the head-space gas chromatographic method as described previously (10).

Determination of LMOX and CMD concentration in plasma and liver: The animals were given the antibiotics subcutaneously at a dose of $1,000 \mathrm{mg} / \mathrm{kg}$ body weight, and 
blood and liver samples from decapitated rats were obtained $30 \mathrm{~min}$ to $5 \mathrm{hr}$ after the treatment. Livers were homogenized in $0.1 \mathrm{M}$ phosphate buffer ( $\mathrm{pH} 7.0)$ using an Ultrax (Kinematika Co., Swiss), and the homogenate was centrifuged at $10,000 \times \mathrm{g}$ for $20 \mathrm{~min}$. The resulting supernatant and plasma samples were used to measure the antibiotic concentration. CMD and LMOX were measured by the band culture bioassay method using Bacillus subtilis ATCC 6633 and Escherichia coli 7437 , respectively (14).

Determination of antibiotics and NMTT in urine: Rats were given intravenously antibiotics at $1.000 \mathrm{mg} / \mathrm{kg}$ body weight, and their urine samples were collected periodically. The urine sample was mixed with an equivolume of $0.1 \mathrm{M}$ phosphate buffer $(\mathrm{pH}$ 6.9), and the resulting sample was further diluted with an equivolume of a mixture of acetonitrile and $2 \mathrm{mM}$ tetra- $n$-butyl ammonium hydroxide (TBA) in $10 \mathrm{mM}$ phosphate buffer, pH $6.5(1: 4, V / v)$. Next the diluted sample was passed through a Millipore column guard (type SJHV. $0.45 \mathrm{~km}$, Millipore Co., MA), and the resulting filtrate was used for the analysis by high performance liquid chromatography (HPLC). HPLC analysis was performed with a Shimadzu LC-6A system equipped with a LC-6A pump, a SIL-6A injector, a SPD-6A detector (set at $254 \mathrm{~nm}$ ) and a SCL-6A system controller. The column was a Nucleosil $5 \mathrm{C}_{18} \quad(4.6 \mathrm{~mm}$ 1.D. $\times 150 \mathrm{~mm}$ ) with a precolumn of $18-\mathrm{GU}$ spheri-10 RP$18(4.6 \mathrm{~mm}$ I.D. $\times 30 \mathrm{~mm}, 10 \mathrm{~km})$. A linear gradient system using "Solution A" (2 mM TBA in $10 \mathrm{mM}$ phosphate buffer. $\mathrm{pH}$ 6.5) and "Solution B" (acetonitrile) as a mobile phase was employed to avoid interferences from the urinary components. The mobile phase (mixtures of solutions $A$ and $B$ ) was changed linearly from 93:7 ( $/ \mathrm{v}$ ) to $75: 25$ $(\mathrm{v} / \mathrm{v})$ over $15 \mathrm{~min}$, and then a $40: 60(\mathrm{v} / \mathrm{v})$ mixture of solutions $A$ and $B$ was passed throgh the column for $2 \mathrm{~min}$. After the washing with a 40:60 ( $/ v / v)$ mixture of solutions $A$ and $B$ for 5 min, the column was equilibrated with a mixture of solutions $A$ and $B(93: 7, v / v)$ for $7 \mathrm{~min}$ for the next use. Under the analytical conditions employed. various beta-lactam antibiotics and NMTT were eluted with $t_{\mathrm{R}}$ values of $18-21$ and 5-6 min, respectively.

Chemicals: The sodium salt of NMTT was prepared in this laboratory. Disulfiram and TBA were purchased from Wako Chemical Industries (Osaka). Other chemicals of the purest grade available were obtained commercially and used for the experiments without further purification.

\section{Results}

\section{Effect of beta-lactam antibiotics on} alcohol-metabolizing system in rats: Blood acetaldehyde levels in rats pretreated with NMTT-containing antibiotics were compared with those of disulfiram-treated rats. Animals were given antibiotics subcutaneously and then given orally ethanol at varying times after the antibiotic-treatments. Blood samples for the determination of acetaldehyde level were obtained $1 \mathrm{hr}$ after the ethanol administration. Blood acetaldehyde level markedly increased when ethanol was administered $18 \mathrm{hr}$ after the antibiotic (Fig. 1). Administration of ethanol 3-5 hr and $48 \mathrm{hr}$ after the antibiotic tended to increase the acetaldehyde level, but only slightly. with all the antibiotics examined (Fig. 1A). Interestingly, administration of disulfiram, a well-known potent inhibitor of Iow $K_{m} \operatorname{ALDH}(15,16)$, caused a similar time course of the effect on the blood acetaldehyde level (Fig. 1B). The effects of antibiotics on liver mitochondrial acetaldehyde-metabolizing enzyme(s) were determined using LMOX and CMD as a model of NMTT-containing antibiotics. The mitochondrial low $K_{x}$ ALDH activity decreased 3-5 hr after antibiotic treatment with the maximal effect coming 5-48 hr after the treatment. The activities recovered to the normal range about 5 days later (Fig. 2A). On the other hand, high $K_{m}$ ALDH activities in mitochondria and microsomes and cytosolic alcohol dehydrogenase activity were not altered by the administration of either LMOX or CMD (data not shown). When the animals were given disulfiram. mitochondrial low $\mathrm{K}_{\mathrm{m}}$ ALDH activity showed a change similar to the cases of antibiotic treatments (Fig. 2A). Acetaldehyde oxidation activity by mitochondrial enzyme(s) 


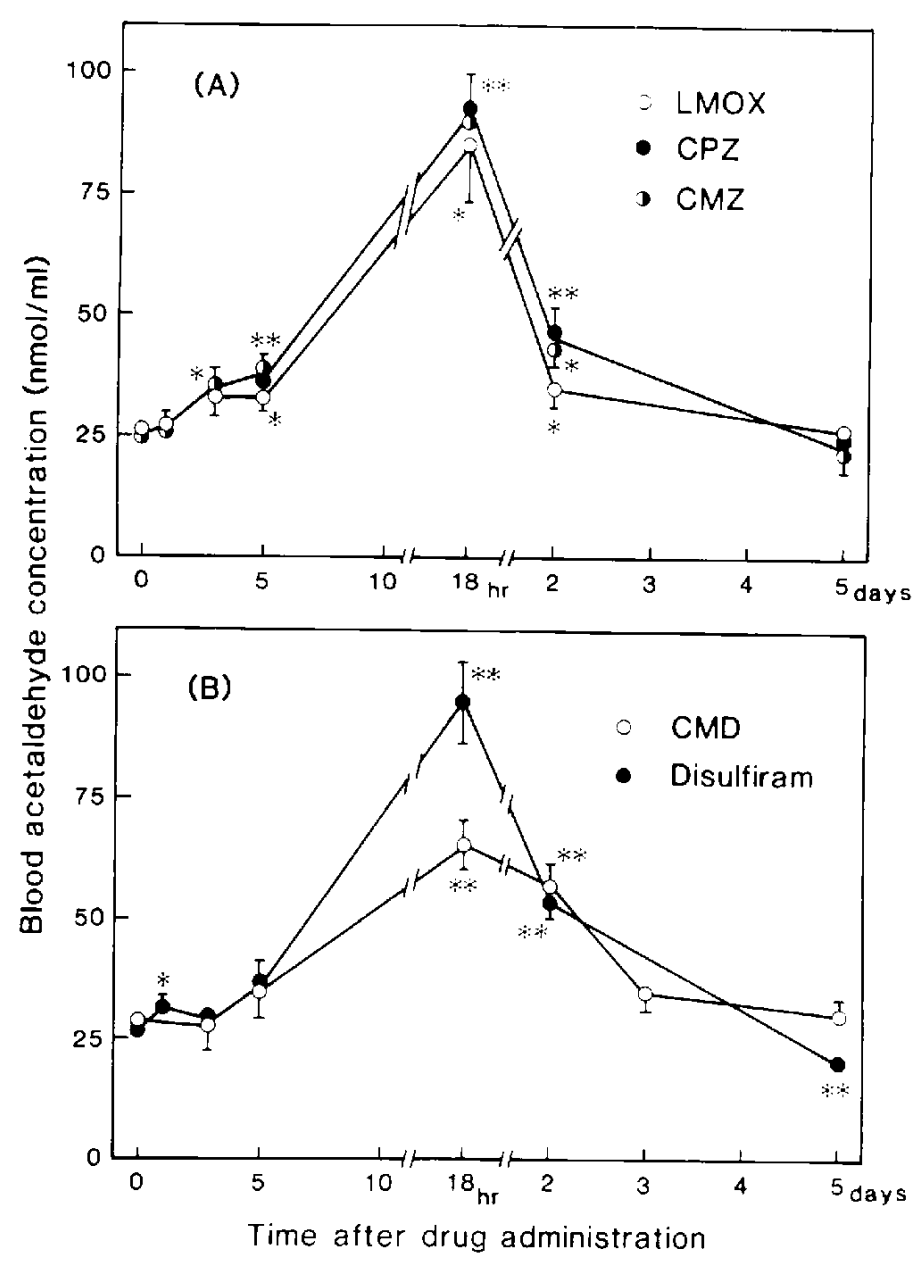

Fig. 1. Time course of the effects of NMTT-containing antibiotics and disulfiram on blood acetaldehyde level during ethanol metabolism. The animals were subcutaneously given antibiotics at a dose of 1.000 $\mathrm{mg} / \mathrm{kg}$ and then orally given ethanol $(2,000 \mathrm{mg} / \mathrm{kg}) 1 \mathrm{hr}$ to 5 days later. In the case of disulfiram treatment, the rats received the compound $(1,000 \mathrm{mg} / \mathrm{kg})$ orally. Blood samples were obtained $1 \mathrm{hr}$ after the ethanol treatment, and blood acetaldehyde concentration was determined and plotted as a function of the time interval between antibiotic and ethanol administrations. Data of CMD and disulfiram treatments (B) from previous reports (8) are referred to. Each value represents the mean and standard error of 5 rats. *. "*: significantly different fram the corresponding control (time " 0 "). $P<0.05$ and $P<0.01$, respectively.

showed a pattern similar to that of low $K_{m}$ ALDH activity, and no difference was obtained between the antibiotic and disulfiram treatments (Fig. 2B). Administration of CMZ or $\mathrm{CPZ}$, beta-lactam antibiotics containing NMTT, also produced the same time course of effects in low $K_{m}$ ALDH activity and blood acetaldehyde level (data not shown). The results indicated that the NMTT-containing antibiotics affected the alcohol-metabolizing system with the same time course of effects as that of disulfiram. No difference was observed in the effects among the NMTTcontaining antibiotics examined.

Concentrations of antibiotics in plasma and liver: Plasma and liver concentrations of antibiotics in rats treated with a high dose of LMOX and CMD were determined by a bioassay method to obtain a correlation between the decrease in liver enzyme activity and liver concentration of antibiotics, although pharmacokinetic studies on both 


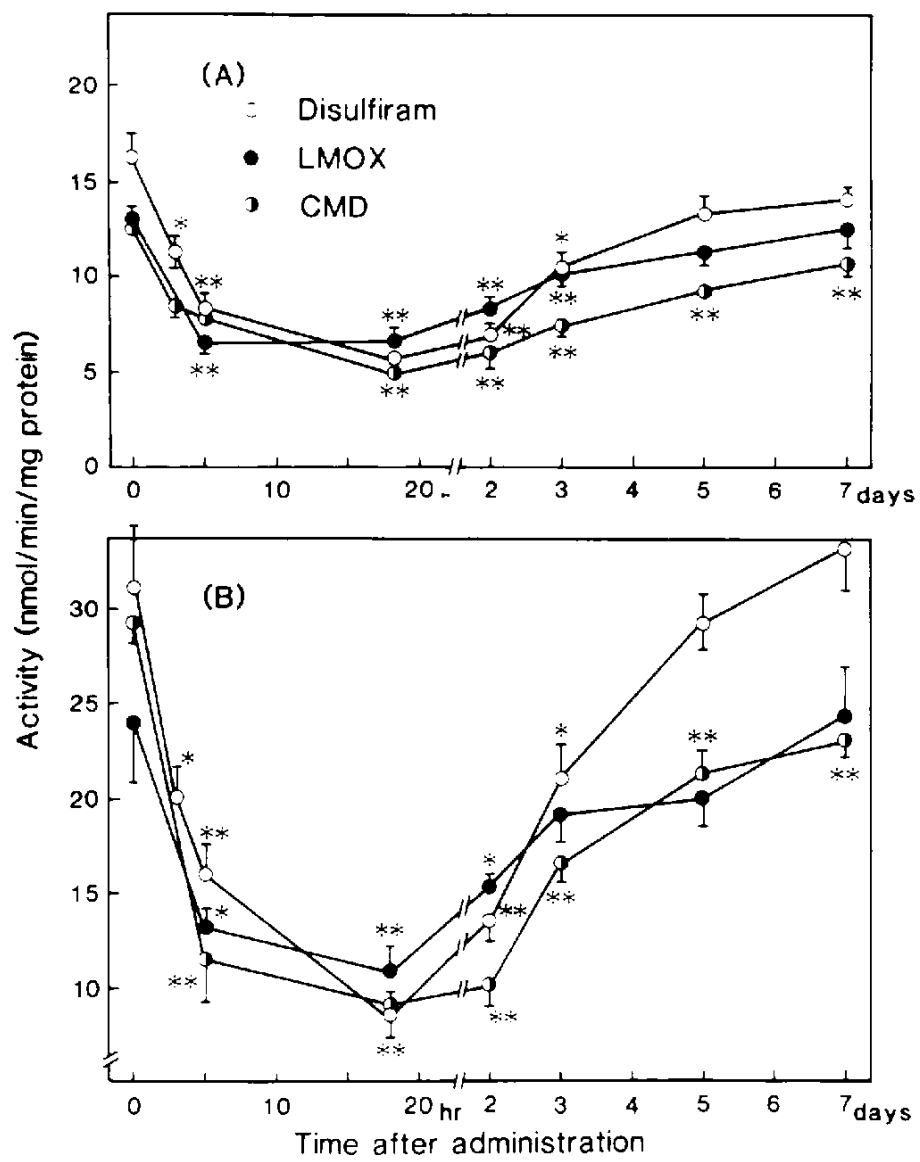

Fig. 2. Alteration of low $\mathrm{K}_{\mathrm{II}} \mathrm{ALDH}$ and acetaldehyde oxidation activities in rat liver mitochondria following administration of antibiotics or disulfiram. Rats received antibiotics $(1,000 \mathrm{mg} / \mathrm{kg}, \mathrm{s} . \mathrm{c}$.), and their liver samples were obtained $3 \mathrm{hr}$ to 7 days after the drug administration. The activities of low $\mathrm{K}_{\mathrm{m}} \mathrm{ALDH}$ (A) and acetaldehyde oxidation (B) in liver mitochondria were determined as reported previously (10). Each value in the figure represents the mean and standard error of 4 animals. * ${ }^{* *}$ : statistically different from the corresponding control, $P<0.05$ and $P<0.01$, respectively.

antibiotics have been carried out in rats administered lower or therapeutic doses of antibiotics (17-20). LMOX and CMD displayed very high plasma levels $30 \mathrm{~min}$ after the treatment, but disappeared rapidly and were undetectable $5 \mathrm{hr}$ later. Liver concentrations of both antibiotics were also higher $30 \mathrm{~min}$ after the injection, although the concentrations were very different between them. Then, the levels fell rapidly. neither could be detected $5 \mathrm{hr}$ after the injection (Fig. 3). The results shown in Figs. 1-3 indicated clearly that the decrease in low $K_{m}$ ALDH activity and the increase in blood acetaldehyde level during ethanol metabolism were followed by a disappearance of the antibiotic from the plasma and the liver.

Effect of beta-lactam antibiotics on mitochondrial low $K_{m}$ ALDH activity in vitro: The direct effect of antibiotics on mitochondrial low $K_{m}$ ALDH activity was checked by adding several antibiotics and disulfiram to the assay mixture. Addition of disulfiram caused concentration-dependent inhibition of the low $K_{m}$ enzyme activity (Fig. 4): about $20 \mu \mathrm{M}$ of disulfiram inhibited the enzyme activity to one-half of the control level. Addition of antibiotics also caused inhibition of the enzyme activity, but the 
(A) LMOX

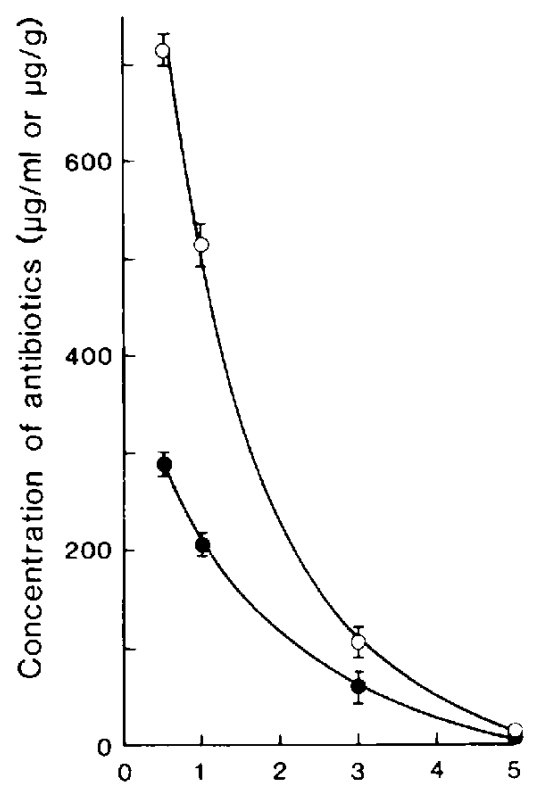

(B) CMD

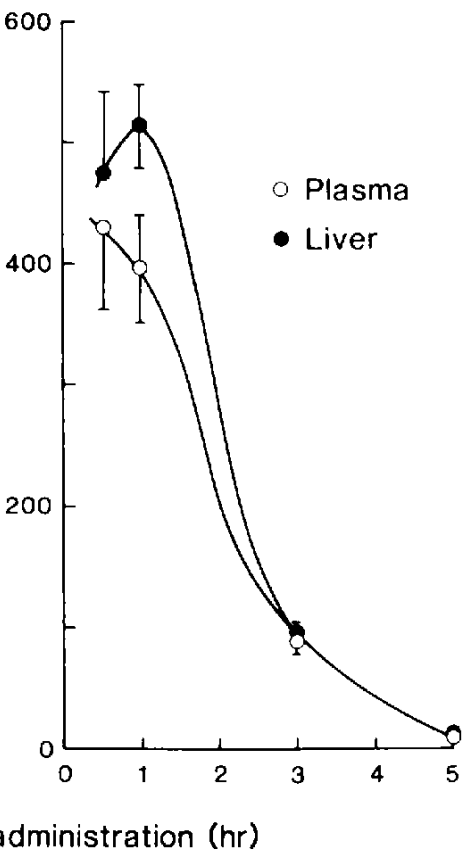

Fig. 3. Plasma and liver concentrations of antibiotics in rats. Rats were given LMOX (A) or CMD (B) subcutaneously at $1,000 \mathrm{mg} / \mathrm{kg}$, and plasma and liver samples were obtained $0.5,1,3$ and $5 \mathrm{hr}$ after the treatment. The concentrations of antibiotics in the samples were determined by the band culture assay method. Each value in the figure represents the mean and standard error of 4 animals.

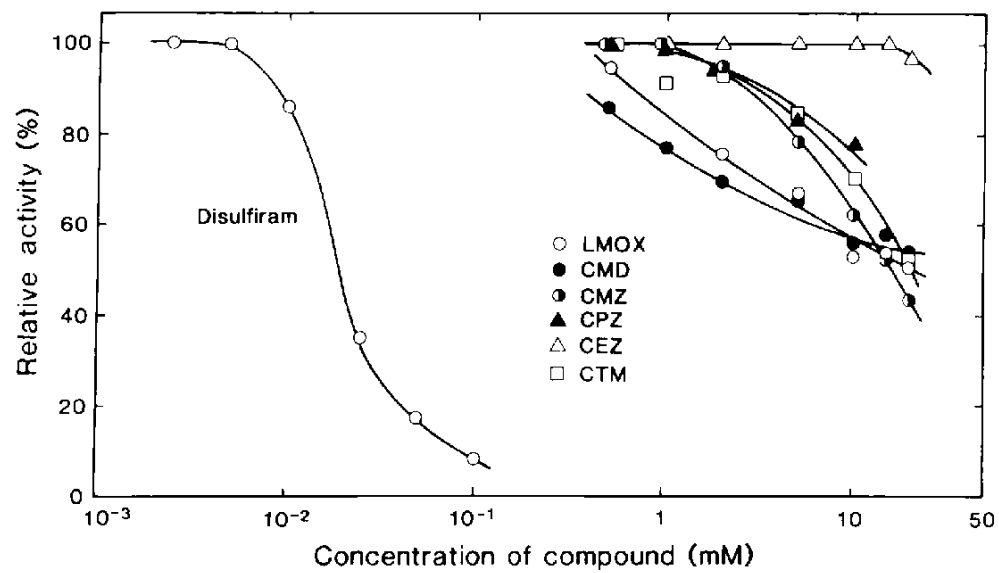

Fig. 4. In vitro effect of disulfiram and antibiotics on liver mitochondrial low $K_{\text {In }}$ ALDH activity. Liver mitochondrial fraction was obtained from intact rats, and the activity was measured at $25^{\circ} \mathrm{C}$ as described previously (10). Mitochondrial concentration in the reaction mixture was about $1 \mathrm{mg}$ protein $/ 3 \mathrm{ml}$ of assay mixture. Enzyme samples were preincubated at $25^{\circ} \mathrm{C}$ for $20 \mathrm{~min}$ with the test compound, and then reaction was initiated by adding $180 \mu \mathrm{M}$ acetaldehyde. The relative enzyme activity obtained in the presence of compounds against the control is plotted as a function of drug concentration. Control enzyme activity was $13.7 \pm 0.7 \mathrm{nmoles} / \mathrm{min} / \mathrm{mg}$ protein $(n=8)$. 
concentration required was $1 \mathrm{mM}$ or more (Fig. 4). Interestingly, all antibiotics tested affected the enzyme activity, although CEZ showed a slight inhibitory effect under the experimental conditions employed. The antibiotic (CTM) having no NMTT in its molecule also caused a concentrationdependent inhibition, while the in vivo administration of CTM caused no alteration in the acetaldehyde-metabolizing system $(9,10)$. The results probably indicated that the in vitro inhibitory effect of antibiotics on the low $K_{m}$ A.LDH activity did not reflect the in vivo effect of antibiotics on the alcoholmetabolizing enzyme system.

Effect of NMTT administration on alcoholmetabolizing system in rats: As described previously (4-10), administration of NMTTcontaining beta-lactam antibiotics and NMTT itself caused an increase in the blood acetaldehyde level during the alcohol metabolism, which was associated with the decrease in liver mitochondrial low $K_{m}$ ALDH activity. Thus, the time course in the effect of NMTT administration was determined and compared with that of antibiotics administration. Remarkable decreases in hepatic low $\mathrm{K}_{\mathrm{m}}$ ALDH and acetaldehyde oxidation activities were observed $3 \mathrm{hr}$ after the NMTT treatment, and the lowest activities were detected continuously for about 2 days. The activities then recovered gradually to the normal range (Fig. 5. A and C). Liver mitochondrial high $K_{m}$ ALDH activity showed almost the same pattern as that of the low $K_{m}$ enzyme, but only a slight decline was obtained (Fig. $5 B$ ). On the other hand. liver alcohol dehydrogenase $(A D H)$ activity was not changed by the administration of NMTT (Fig. 5D). When ethanol was administrered to NMTTpretreated animals, the blood acetaldehyde level increased markedly associated with the decrease in low $K_{m}$ ALDH activity as shown in Fig. 5 . The highest concentration

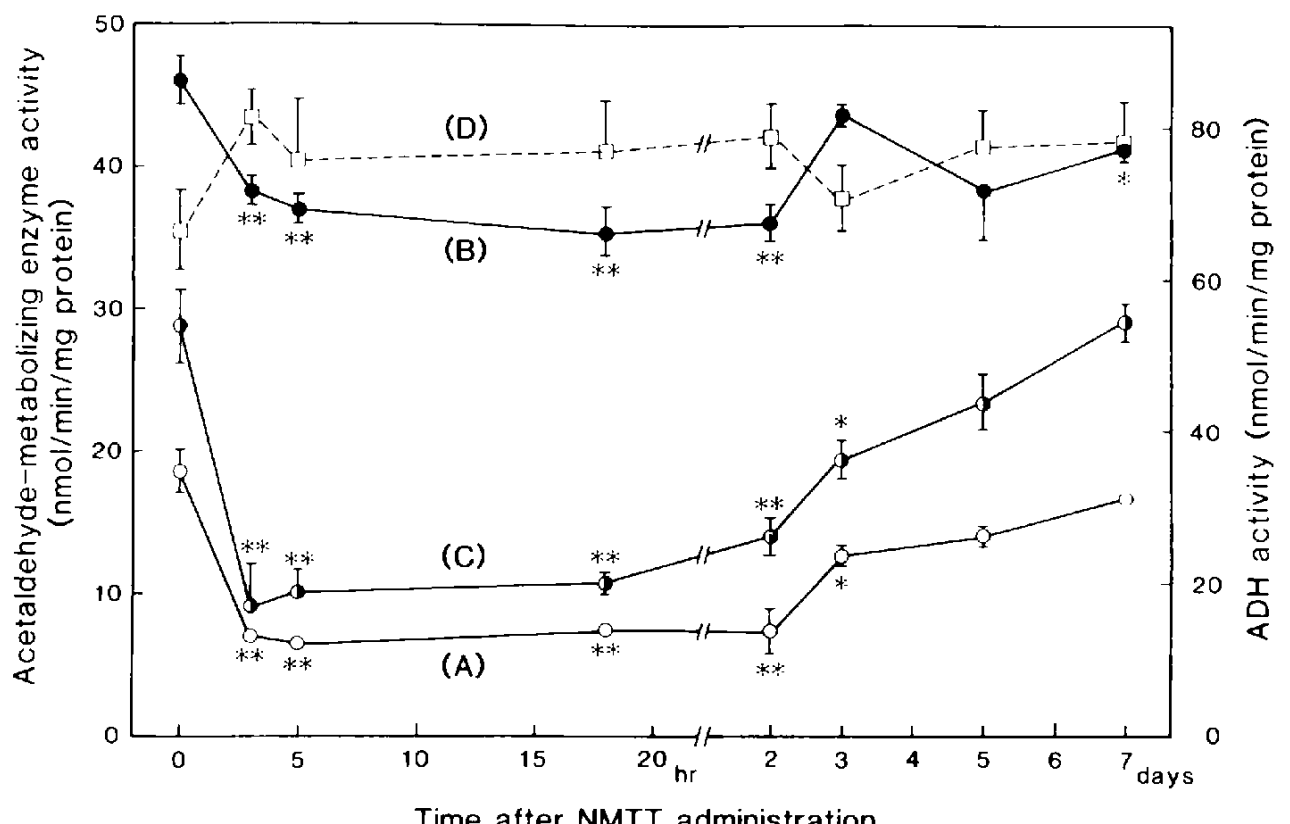

Fig. 5. Alteration of hepatic alcohol-metabolizing enzyme activities in rats following administration of NMTT. The animals were subcutaneously given the sodium salt of NMTT at a dose of $500 \mathrm{mg} \mathrm{NMTT/kg}$ (750 mg NMTT-Na/ $\mathrm{kg}$ ), and liver samples were obtained 3 hr to 7 days after the treatment. The activities of low $K_{m}$ ALDH (A), high $K_{m} A L D H ~(B)$ and acetaldehyde oxidation (C) were measured using isolated liver mitochondria. ADH activity (D) was estimated using a postmitochondrial fraction as an enzyme source. Each value in the figure represents the mean and standard error of 4 animals. * **: statistically significant ( $P<0.05$ and $P<0.01$, respectively) against the corresponding control. 
was observed when ethanol was administered $3-5 \mathrm{hr}$ after the NMTT treatment (Fig. 6 ). The elevated blood acetaldehyde level during the ethanol metabolism fell rapidly. and ethanol administration $18 \mathrm{hr}$ after the NMTT treatment caused only a slight increase in the acetaldehyde level. On the other hand, the blood ethanol level was not affected by NMTT administration (Fig. 6). The results shown in Figs. 5 and 6 demonstrated that NMTT affected the alcohol metabolizing system in vivo similarly to NMTT-containing antibiotics and disulfiram. but the onset of the effect was much more rapid with NMTT.

Urinary excretion of antibiotics and NMTT: To determine the release of NMTT from the parent antibiotics in the body. urinary excretion of the unchanged form of antibiotics and NMTT liberated was measured in rats after intravenous administration of various NMTT-containing antibiotics. The unchanged form of the antibiotics was excreted rapidly, and the excretion was completed within $2 \mathrm{hr}$ after the administration. On the other hand. NMTT was excreted slowly, and the excretion was observed continuously at $6 \mathrm{hr}$ after the treatment, although the amounts of NMTT excreted was very low compared with those of the unchanged form (Table 1). Cumulative amounts of the unchanged form of the antibiotics excreted into the $0-6 \mathrm{hr}$ urine varied markedly from 58 to $87 \%$ of the dose depending on the antibiotic injected, while the amounts of NMTT liberated were $4-7 \%$ of the dose (Table 1). The results indicated that almost similar of NMTT were liberated in the body from various NMTT-containing antibiotics.

In vitro effect of NMTT on mitochondrial low $\mathrm{K}_{\mathrm{m}}$ ALDH: As mentioned above. NMTT released from its parent antibiotics was supposed to inhibit liver low $K_{m}$ ALDH activity followed by the development of disulfiram-like effects. Then, the effect of NMTT on liver mitochondrial low $K_{m}$ ALDH activity was studied in vitro in comparison with that of disulfiram. When intact mitochondria were used as the enzyme source. only high $K_{m}$ $A L D H$ activity, but not low $K_{\text {ru }}$ enzyme activity. was detected. Following the solubilization of enzymes by mixing the mitochondrial

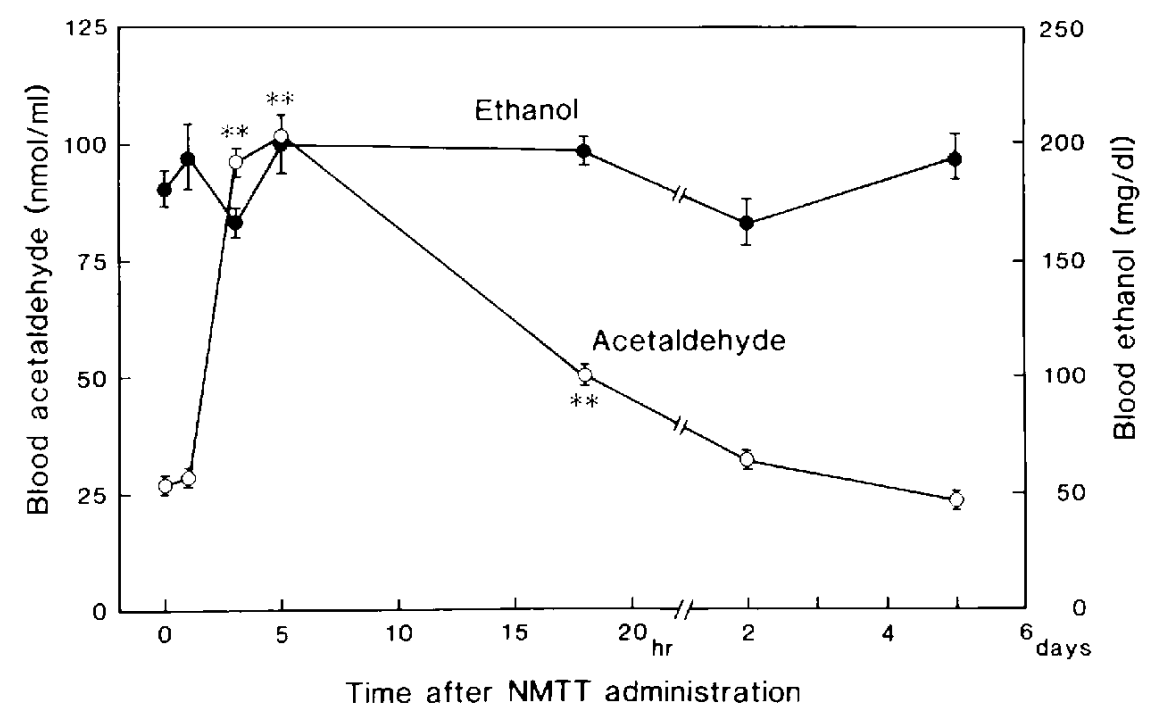

Fig. 6. Blood levels of acetaldehyde and ethanol in the NMTT-pretreated rats during ethanol metabolism. The animals were given subcutaneous administration of the sodium salt of NMTT at a dose of $300 \mathrm{mg}$ NMTT $/ \mathrm{kg}$. Next, ethanol $(2,000 \mathrm{mg} / \mathrm{kg})$ was administered orally $1 \mathrm{hr}$ to 5 days after the NMTT treatment as shown in the figure. Blood samples were obtained 1 hr after the administration of ethanol. Each value in the figure represents the mean and standard error of 5 rats. ** : statistically significant $(P<0.01)$ against the control. 
Table 1. Cumulative urinary excretion of antibiotics and NMTT after intravenous administration

\begin{tabular}{|c|c|c|c|c|c|c|}
\hline \multirow{2}{*}{ Antibiotic } & \multirow{2}{*}{$\begin{array}{l}\text { Excreted } \\
\text { form }\end{array}$} & \multicolumn{5}{|c|}{ Cumulative excretion ( $\%$ of dose) } \\
\hline & & $0-0.5 \mathrm{hr}$ & $0.5-1.0 \mathrm{hr}$ & $1.0-2.0 \mathrm{hr}$ & $2.0-4.0 \mathrm{hr}$ & $4.0-6.0 \mathrm{hr}$ \\
\hline \multirow[t]{2}{*}{ Latamoxef } & Unchanged form & $55.3 \pm 2.4$ & $75.1 \pm 1.4$ & $84.8 \pm 0.9$ & $87.0 \pm 0.7$ & $87.2 \pm 0.6$ \\
\hline & NMTT & $1.1 \pm 0.1$ & $2.2 \pm 0.1$ & $4.0 \pm 0.1$ & $5.9 \pm 0.2$ & $6.7 \pm 0.2$ \\
\hline \multirow[t]{2}{*}{ Cefamandole } & Unchanged form & $56.7 \pm 2.3$ & $72.3 \pm 0.9$ & $75.7 \pm 0.9$ & $76.3 \pm 1.0$ & $76.4 \pm 1.0$ \\
\hline & NMTT & $1.3 \pm 0.2$ & $2.3 \pm 0.2$ & $3.2 \pm 0.3$ & $4.2 \pm 0.2$ & $5.0 \pm 0.3$ \\
\hline \multirow[t]{2}{*}{ Cefoperazone } & Unchanged form & $46.2 \pm 1.0$ & $55.9 \pm 1.6$ & $57.7 \pm 1.6$ & $57.9 \pm 1.7$ & $58.0 \pm 1.7$ \\
\hline & NMTT & $3.1 \pm 0.1$ & $4.7 \pm 0.03$ & $5.7 \pm 0.2$ & $6.7 \pm 0.1$ & $7.4 \pm 0.2$ \\
\hline \multirow[t]{2}{*}{ Cefmetazole } & Unchanged form & $58.3 \pm 1.0$ & $66.0 \pm 1.6$ & $68.4 \pm 2.0$ & $68.5 \pm 2.0$ & $68.6 \pm 2.0$ \\
\hline & NMTT & $2.9 \pm 0.1$ & $4.1 \pm 0.2$ & $5.4 \pm 0.2$ & $6.0 \pm 0.2$ & $6.9 \pm 0.2$ \\
\hline \multirow[t]{2}{*}{ Cefmenoxime } & Unchanged form & $55.7 \pm 1.4$ & $70.1 \pm 1.8$ & $77.3 \pm 1.5$ & $79.1 \pm 1.5$ & $79.5 \pm 1.5$ \\
\hline & NMTT & $1.4 \pm 0.1$ & $2.2 \pm 0.2$ & $2.9 \pm 0.2$ & $3.5 \pm 0.2$ & $4.1 \pm 0.2$ \\
\hline \multirow[t]{2}{*}{ Cefotetan } & Unchanged form & $47.2 \pm 1.2$ & $63.0 \pm 2.0$ & $71.5 \pm 2.2$ & $75.0 \pm 3.0$ & $75.8 \pm 3.0$ \\
\hline & NMTT & $2.2 \pm 0.1$ & $3.5 \pm 0.2$ & $5.1 \pm 0.1$ & $6.2 \pm 0.1$ & $6.9 \pm 0.2$ \\
\hline
\end{tabular}

Rats were given intravenously various antibiotics at $1.000 \mathrm{mg} / \mathrm{kg}$, and their urine samples were collected periodically. Amounts of NMTT excreted were calculated as those of antibiotics. The values in the table represent the mean $\pm S$.E. of 4 rats.

samples with a detergent, deoxycholate, for about $5 \mathrm{~min}$ at $25^{\circ} \mathrm{C}$, we could detect both high and low $\mathrm{K}_{\mathrm{m}}$ ALDH activities. Then, the effects of NMTT and disulfiram on mitochondrial low $\mathrm{K}_{\mathrm{m}}$ enzyme activity was determined by adding these compounds to the solubilized system. As shown in Fig. 7. addition of disulfiram resulted in a decrease in the enzyme activity depending on the preincubation or solubilization time. while addition of NMTT caused a slight effect on the ALDH activity. On the contrary, the administration of NMTT to rats caused a marked inhibition of mitochondrial enzyme (Fig. 5). Direct inhibitory action of NMTT on mitochondrial enzyme was not observed even after the preincubation for $60 \mathrm{~min}$. Disufiram showed, on the other hand, the inhibitory action in both in vivo and in vitro systems. Then, the effects of disulfiram and its related compound on the acetaldehydemetabolizing system were determined to obatain the in vivo and in vitro correlation in the enzyme inhibition. Administration of disulfiram (tetraethyl-thiuram disulfide) and its monosulfide form (diethyldithiocarbamate) resulted in decreases in mitochondrial low $\mathrm{K}_{\mathrm{m}} \mathrm{ALDH}$ and acetaldehyde oxidation activities in the liver, and the blood acetaldehyde level increased markedly when the

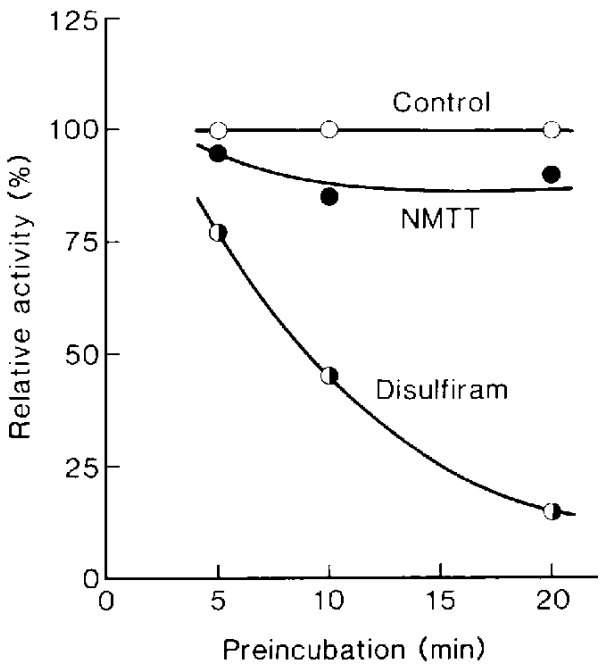

Fig. 7. In vitro effects of disulfiram and NMTT on mitochondrial low $K_{m}$ ALDH activity. Rat liver mitochondria were suspended in $2.9 \mathrm{ml}$ of the incubation mixture containing $50 \mathrm{mM}$ pyrophosphate buffer $(\mathrm{pH} 8.5), 0.5 \mathrm{mM}$ NAD, $2 \mu \mathrm{M}$ rotenone. 0.1 $\mathrm{mM}$ pyrazole and $0.5 \mathrm{mg}$ deoxycholate, and the mixture was preincubated at $25^{\circ} \mathrm{C}$ for $5-20$ min as shown in the figure. Then, the reaction was initiated by adding $0.1 \mathrm{ml}$ acetaldehyde (180 $\mu \mathrm{M}$ at final), and the enzyme reaction was monitored by the absorbance change at $340 \mathrm{~nm}$. Disulfiram (in acetone) and sodium salt of NMTT (in water) were added to the mixture at the final concentrations of 0.1 and $5 \mathrm{mM}$. respectively, just prior to the preincubation. Mitochondrial concentration was $0.33 \mathrm{mg}$ protein $/ \mathrm{ml}$. 
animals were given ethanol $18 \mathrm{hr}$ after the disulfiram and its monosulfide form treatments. When disulfiram and diethyldithiocarbamate were added to the ALDH assay system, a pronounced inhibition of mitochondrial low $K_{m}$ enzyme activity was detected by disulfiram, as shown in Figs. 4 and 7 , while no alteration in the enzyme activity was observed by diethyldithiocabamate even by the addition of a high concentration ( $5 \mathrm{mM}$ ) (data not shown). These results indicated probably that the disulfide linkage of the compound is important in the manifestation of its inhibitory action on low $\mathrm{K}_{\mathrm{m}}$ ALDH.

\section{Discussion}

Beta-lactam antibiotics, having the $\mathrm{N}$ methyltetrazolylthiomethyl group at the 3position of the cephalosporin nucleus, display a disulfiram-like reaction, which is detected in rats as a decrease in low $K_{n}$ ALDH and acetaldehyde oxidation activities and an increase in blood acetaldehyde level during ethanol metabolism (3-10). Timecourse studies on the effect of several NMTT-containing antibiotics upon the alcohol-metabolizing system indicated the similarities of all antibiotics examined in producing a disulfiram-like effect (Figs. 1 and 2). Interestingly, blood acetaldehyde level increased during ethanol metabolism in all NMTT-containing antibiotics examined when mitochondrial low $\mathrm{K}_{\mathrm{m}}$ ALDH activity showed the lowest values (Figs. 1 and 2). The results indicate that a decrease in the low $K_{m}$ enzyme activity causes a decrease of acetaldehyde oxidation activity followed by an increase in blood acetaldehyde level during ethanol metabolism. However, some discrepancies were observed between the low $K_{m}$ ALDH activity and blood acetaldehyde level, as pointed out previously (10); depression of the enzyme activity was observed for a longer time compared with the increase in blood acetaldehyde level. Thus, this is still the subject for further study.

Yamanaka et al. (9) demonstrated the in vitro inhibition of mitochondrial low $\mathrm{K}_{\mathrm{m}}$ ALDH activity by the NMTT-containing antibiotics and suggested a similarity in the action mechanism of antibiotics between the in vitro and in vivo system. As shown in Fig. 4, the inhibitory effect of various antibiotics on mitochondrial low $K_{m}$ enzyme activity in vitro was, however, observed by adding $1 \mathrm{mM}$ or a much higher concentration (about $500 \mu \mathrm{g} / \mathrm{ml}$ or more) regardless of whether or not NMTT was present in their molecules. In addition. NMTT-containing antibiotics, LMOX and CMD, were detected in rat plasma and liver only for about several hours after the treatment, even after the administration of a high dose $(1.000 \mathrm{mg} / \mathrm{kg})$ of the antibiotics, and the concentrations of LMOX and CMD in liver were $287 \pm 13$ and $47 \pm 69 \mu \mathrm{g} / \mathrm{g}$ liver $(n=4)$, respectively, $30 \mathrm{~min}$ after the treatments (Fig. 3). If the whole molecular structure of the antibiotic had participated in the development of the disulfiram-like reaction, the maximal effect should have developed soon after the treatment, but this did not occur, as can be seen from Figs. 1 and 2. The results suggest that the in vitro inhibitory action of antibiotics is not correlated with the in vivo disulfiram-like effect of these drugs.

NMTT is a common 3'-position substituent of the cephalosporin nucleus of betalactam antibiotics, which display a disulfiram-like reaction $(2-10,21)$. Administration of NMTT resulted in a rapid onset of the disulfiram-like reaction in rats (Figs. 5 and 6 ). Rapid onset of the effect of NMTT has also been reported when the indicators were the increase in blood acetaldehyde level and the decreased metabolic conversion of alcohol to $\mathrm{CO}_{2}(4-10,21,22)$. All these results suggest that the disulfiram-like reaction associated with beta-lactam antibiotics is mediated by NMTT which is released from the parent drugs. The occurrence of this release has been demonstrated with rats and humans (23-25), and thus the pharmacokinetic profiles of antibiotics and NMTT liberated should be important for understanding the development of disulfiram-like effects of parenteral betalactam antibiotics. Yanagihara et al. (8) and Turcan et al. (21) demonstrated the correlation between the biliary excretion of antibiotics and the development of their disulfiram-like effects. We demonstrated also the excretion of antibiotics into bile followed 
by the degradation of antibiotics and the release of NMTT in the bile duct and/or intestine at alkaline and acidic $\mathrm{pH}$ without any participation of intestinal bacteria (26). The NMTT liberated was absorbed completely from intestine which caused NMTT to remain in the body at high levels for a long period with the development of the disulfiram-like effects $(27,28)$. Pronounced disulfiram-like effects of NMTT-containing antibiotics were observed by the oral administration of drugs. when compared with the intravenous and subcutaneous administrations. When the antibiotic was given orally, higher concentrations of NMTT, but not the parent antibiotic. were detected in plasma and liver for a long time. On the other hand, intravenous administration of the NMTT-containing antibiotics to biliary fistula rats caused no development of disulfiram-like effects concomitant with a rapid disappearance of NMTT from the body (27). All these results indicated that the NMTT liberated from its parent drugs, but not the whole molecular structure of betalactam antibiotics, produces the disulfiramlike effects. When various NMTT-containing antibiotics were administered intravenously. almost the same amounts of NMTT were excreted into the urine (Table 1). indicating almost the same capability to develop the disulfiram-like effects in these antibiotics. In the previous paper (10), we checked the dose-activity relationship in the increase of blood acetaldehyde level, which indicated the similarity among the NMTT-containing antibiotics examined. These results are consistent with the above assumption.

Unfortunately, we failed to show the direct inhibitory action of NMTT on low $K_{m}$ ALDH in vitro, although administration of NMTT resulted in the development of disulfiram-like effects (Figs. 5-7). Similar results were obtained when diethyldithiocarbamate, a monosulfide form of disulfiram. was used instead of NMTT. Disulfiram is known to be reduced rapidly to diethyldithiocarbamate by the interaction with plasma proteins $(29,30)$. Ineffectiveness of diethyldithiocarbamate to inhibit ALDH activity was also reported by others $(31,32)$. The results indicated probably that diethyl- dithiocarbamate undergoes some activation step in vivo which enables it to interact with low $K_{m}$ ALDH. Several investigators suggested the reaction between the thiol group of the active site of low $K_{m}$ ALDH and disulfiram or diethyldithiocarbamate to show the enzyme inhibition $(33,34)$. The discrepancy in the inhibitory action of NMTT between in vivo and in vitro systems may be explainable as described above even in the case of NMTT.

In conclusion, our time-course studies on the effects of NMTT-containing antibiotics and NMTT itself upon the ethanolmetabolizing system in rats demonstrated a close correlation between the decrease of low $K_{m}$ ALDH activity and the increase in blood acetaldehyde level during ethanol metabolism. Maximal effects of antibiotics and NMTT, as detected by blood acetaldehyde levels, were observed 18 and 5 hr. respectively. after the administration. Although various antibiotics inhibited mitochondrial low $K_{\mathrm{m}}$ ALDH activity in vitro at 1 $\mathrm{mM}$ or more, such a high concentration of antibiotics in the liver was detected only soon after their administration. Therefore, the whole molecular structure of the NMTTcontaining antibiotics was not associated with the development of the disulfiram-like reaction. On the other hand, administration of NMTT itself caused a rapid onset of the effect on the alcohol-metabolizing system, indicating that NMTT released from the parent antibiotic is associated with the disulfiram-like reaction of beta-lactam antibiotics

Acknowledgment: The authors thank Dr. $T$. Yoshizaki, Messrs, Y. Nishibe, H. Fujitomo and $Y$. Kimura, and Miss K. Fujimoto of this laboratory for their technical assistance.

\section{References}

1 Ritchie, J.M.: The aliphatic alcohols. In The Pharmacological Basis of Therapeutics, Edited by Goodman, L.S. and Gilman, A., Fifth edition. p. 137-151. MacMillan Publishing Co., New York (1975)

2 Foster, T.S., Raell, C.L. and Wilson, H.D.: Disulfiram-like reaction associated with a parenteral cephalosporin. Am. J. Hosp. Pharm. $37,858-859(1980)$ 
3 Buening, M.K., Wold, J.S., Israel, K.S, and Kammer, R.B.: Disulfiram-like reaction to betalactams. JAMA 245, 2027-2028 (1980)

4 Buening, M.K. and Wold, J.S.: Ethanolmoxalactam interactions in vivo. Rev. Infect. Dis. 4. Supp. S555-S563 (1982)

5 Uri, J.V. and Parks, D.B.: Disulfiram-like reaction to cephalosporins. Ther. Drug Monit. 5, 219-224 (1983)

6 Brien, J.F, and Loomis, C.W.: Aldehyde dehydrogenase inhibitors as alcohol-sensitizing drugs: a pharmacological perspective. Trends Pharmacol. Sci. 6, 477-480 (1985)

7 Yanagihara, M., Okada, K., Nozaki, M., Tsurumi, K. and Fujimura, H.: Cephem antibiotics and alcohol metabolism. (1) Disulfiram-like reaction resulting from intravenous administration of cephem antibiotics. Folia Pharmacol. Japon. 79, 551-560 (1982) (Abs. in English)

8 Yanagihara, M., Okada, K., Nozaki, M., Tsurumi, K. and Fujimura, H.: Cephem antibiotics and alcohol metabolism. Japan. J. Antibiotics 38 , 634-642 (1985)

9 Yamanaka, Y., Yamamoto, T. and Egashira, T.: Effects of cephem antibiotics on rat liver aldehyde dehydrogenases. Japan. J. Pharmacol. 33, 717-723 (1983)

10 Matsubara, T., Otsubo, S., Ogawa, A., Okamoto, J., Yoshizaki, T., Nishibe, Y., Tochino, Y. and Hirai, E.: A comparative study on the effects of disulfiram and beta-lactam antibiotics on the acetaldehyde-metabolizing system in rats. Japan. J. Pharmacol. 42, 333-343 (1986)

11 Lowry, O.H., Rosebrough, N.J., Farr, A.L. and Randall, R.J.: Protein measurement with the Folin phenol reagent. J. Biol. Chem. 193, 265-275 (1951)

12 Hasumura, Y., Teschke, R. and Lieber, C.S.: Characteristics of acetaldehyde oxidation in rat liver mitochondria. J. Biol. Chem. 251, 49084913 (1976)

13 Büttner, $\mathrm{H}$.: Aldehyde- und Alkohol-dehydrogenase Aktivität in Leber und Niere der Ratte. Biochem. Z. 341, 300-314 (1965)

14 Kimura, Y. and Yoshida, T.: Microbiological assay methods of 6059-S concentrations in body fluids. Chemotherapy (Tokyo) 28 (S-7), 178188 (1980) (Abs. in English)

15 Li, T.L.: Enzymology of human alcohol metabolism. Adv. Enzymol. 45, 427-483 (1977)

16 Weiner, H.: Aldehyde oxidizing enzymes. In Enzymatic Basis of Detoxication. Edited by Jakoby. W.B., Vol. 1, p. 261-280, Academic Press, New York (1980)

17 Yoshida, T., Kimura, Y., Doi, M., Nakashimizu,
H. and Tochino, Y.: Pharmacodynamics of cefamandole in experimental animals. Chemotherapy 27 (Tokyo) (S-5), 112-119 (1979) (Abs. in English)

18 Tokiwa, T., Uda, F., Fujino, A. and Tochino, Y.: Absorption, excretion, distribution and metabolism of ${ }^{14} \mathrm{C}$-cefamandole. Chemotherapy (Tokyo) 27 (S-5), 120-134 (1979) (Abs. in English)

19 Yoshida, T., Kimura, Y. and Tochino, Y.: Pharmacokinetics of 6059-S in experimental animals. Chemotherapy (Tokyo) 28 (S-7), 194206 (1980) (Abs. in English)

20 Sugeno, K., Okabe, H., Tanaka, H. and Norikura, R.: Disposition of 6059-S in rats, dogs and monkeys. Chemotherapy (Tokyo) 28 (S-7), 207235 (1980) (Abs. in English)

21 Turcan, R.G., MacDonald, C.M., Ings, R.M.J. and Coombes, J.D.: Inhibition of the rate of ${ }^{14} \mathrm{CO}_{2}$ production from $\left[{ }^{14} \mathrm{C}\right]$ ethanol in rats given beta-lactam antibiotics with disulfiram-like effects. Antimicrob. Agent Chemother. 27, 535540 (1985)

22 Brien, J.F., Tam, G.S., Cameron, R.J., Steenaart, N.A.E. and Loomis, C.W.: A comparative study of the inhibition of hepatic aldehyde dehydrogenases in the rat by methyltetrazolethiol, calcium carbimide, and disulfiram. Can. J. Physiol. Pharmacol. 63, 438-443 (1985)

23 Shindo, H.: Absorption, distribution, excretion and metabolism of cefmetazole. Sankyo Kenkyusho Nempo 31, 42-48 (1979)

24 Nakamura, K., Nakagawa, A., Tanaka, M., Masuda, H., Hayashi, Y. and Saionji, K.: Effects of cephem antibiotics on ethanol metabolism. Folia Pharmacol. Japon. 83, 183-191 (1984) (Abs. in English)

25 Uchida, K., Konishi, M., Akiyoshi, T., Igimi, H. and Asakawa, S.: Biliary excretion of latamoxef and $\mathrm{N}$-methyltetrazolethiol in humans and rats. J. Pharmacobiodyn. 8, 981-988 (1985)

26 Matsubara, T., Otsubo, S., Ogawa, A. and Nakao, H.: Effects of beta-lactam antibiotics on acetaldehyde-metabolizing system in germ-free rats. Japan. J. Pharmacol. 45, 115-119 (1987)

27 Matsubara, T., Otsubo, S., Ogawa, A. and Oka, T.: Pharmacokinetics of latamoxef and $\mathrm{N}$-methyltetrazolethiol in rats associated with the development of disulfiram-like effects. Japan. J. Pharmacol. 45, 223--232 (1987)

28 Matsubara, T., Otsubo, S. and Ogawa, A.: Effect of 6315-S (flomoxef) on the alcoholmetabolizing system in rats. Chemotherapy (Tokyo) 35 (S-1), 460-469 (1987) (Abs. in English)

29 Eneanya, D.I., Bianchine, J.R., Duran, D.O. and 
Andresen, B.D.: The actions and metabolic fate of disulfiram. Annu. Rev. Pharmacol. Toxical. 21, 575-596 (1981)

30 Agarwal, R.P., McPherson, R.A. and Phillips, M.: Rapid degradation of disulfiram by serum protein. Res. Commun. Chem. Pathol. Pharmacol. 42, 293-310 (1983)

31 Dietrich, R. and Erwin, V.: Mechanism of the inhibition of aldehyde dehydrogenase in vivo by disulfiram and diethyl-dithiocarbamate. Mol. Pharmacol. 7, 301-307 (1971)

32 Kitson, T.M.: The effect of disulfiram on the aldehyde dehydrogenase of sheep liver. Biochem.
J. 151, 407-412 (1975)

33 Marchner, $\mathrm{H}$. and Tottmer, $\mathrm{O}$ : Studies in vitro on the inactivation of mitochondrial rat-liver aldehyde dehydrogenase by the alcoholsensitizing compounds cyanamide, 1-aminocyclopropanol and disulfiram. Biochem. Pharmacol. 32, 2181-2188 (1983)

34 Kitson, T.M.: The time course of the interaction of sheep liver cytoplasmic aldehyde dehydrogenase with $2,2^{\prime}$ - and 4,4'-dithiopyridine: a comparison with the action of disulfiram. Arch. Biochem. Biophys. 234, 487-496 (1984) 